\title{
Incidence and predictors of radial artery injury following transradial procedures: Yet another benefit of renin-angiotensin system blockade?
}

\author{
Burak Turan ${ }^{1}$, Ayhan Erkol ${ }^{1}$, Fatih Yilmaz ${ }^{2}$, Mehmet Mustafa Can ${ }^{3}$, İsmail Erden ${ }^{1}$ \\ ${ }^{1}$ Department of Cardiology, Kocaeli Derince Training and Research Hospital, Kocaeli, Turkey \\ ${ }^{2}$ Department of Cardiology, Kartal Kosuyolu Heart Training and Research Hospital, Istanbul, Turkey \\ ${ }^{3}$ Department of Cardiology, Haseki Training and Research Hospital, Istanbul, Turkey
}

\begin{abstract}
Background: Vasodilatory function of radial artery $(R A)$ declines following the transradial catheterization. However, it is uncertain whether impaired vasodilatory function develops in every patient. The aim of this study was to investigate the incidence and predictive factors of impaired vasodilatory function following transradial procedures.
\end{abstract}

Methods: Consecutive patients undergoing elective transradial procedures were prospectively enrolled. Ultrasound examination of $R A$ was recorded just before and 1 week after the procedure. RA diameters and flow velocities were measured at baseline, after flow mediated vasodilation (FMD) and after nitrate mediated vasodilation (NMD).

Results: Fifty-one patients were included (62 \pm 11 years, 55\% male, 41\% hypertensive, $20 \%$ diabetic, 65\% with coronary artery disease). Overall FMD and NMD were significantly impaired after 1 week. However, deterioration of FMD and NMD was observed in $67 \%$ and $71 \%$ of patients, respectively. Absolute change in FMD was significantly different in patients using a renin-angiotensin system (RAS) inhibitor compared to those who were not $(1.9 \pm 12.9$ vs. $-7.7 \pm$ $\pm 12.7 \%$, respectively, $p=0.025)$. Additionally, there was a moderate but significant correlation between baseline RA diameter and absolute change in NMD $(r=0.419, p<0.001)$. RAS blockade was independently associated with protection against FMD deterioration (OR 0.241, 95\% CI 0.066-0.883, $p=0.032$ ), whereas RA diameter (OR 0.079, 95\% CI 0.009-0.720, $p=0.024)$ and procedure time (OR 1.156, 95\% CI 0.989-1.350, $p=0.068$ ) were associated with NMD deterioration, although the latter had borderline significance.

Conclusions: Vasodilatory function of $R A$ gets impaired in most patients following transradial procedures. RAS blockade seems to exert a protective role against deteriorating endothelium-dependent vasodilation, whereas smaller $R A$ diameter and potentially longer procedure time are associated with impaired endothelium-independent vasodilation. (Cardiol J 2016; 23, 1: 64-70)

Key words: radial artery, catheterization, endothelium, vasodilation, renin-angiotensin system

Address for correspondence: Burak Turan, MD, Turgut mah. Hayat cad. Tokuştepe sok. No: 103/7, 41200, İzmit, Kocaeli, Turkey, tel:+90 2623178000 (work), +90 5056293509 (mobile), fax: +90 262233 5536, e-mail: drburakturan@gmail.com 


\section{Introduction}

Transradial approach improves patient comfort and clinical outcomes [1]. However, it is associated with morphological and functional changes in radial artery (RA) which may lead to occlusion. Previous studies with intravascular ultrasound, optical coherence tomography and histological examination revealed that intimal hyperplasia or intima-media thickening, peri-arterial tissue or fat necrosis and adventitial inflammation was evident in previously catheterized RA [2-6]. Vasodilator function of RA following transradial procedures was studied extensively as well, most studies reporting a decline in endothelium-dependent or independent vasodilation in acute phase followed by some recovery in long-term [6-13]. It is uncertain, however, whether vasodilatory function gets impaired in every patient or not. Therefore, the aim of this study was to find out the incidence and predictive factors of impaired vasodilatory function following transradial procedures.

\section{Methods}

The study was a single-center longitudinal observational trial. Consecutive patients over 18 years old undergoing elective transradial procedure were prospectively enrolled. Exclusion criteria were i) emergency procedures, ii) patients with previous a procedure from the same RA (if contralateral RA cannot be used), iii) patients unwilling to participate. The study was conducted according to the Declaration of Helsinki - ethical principles for medical research involving human subjects [14]. All patients participating in the study gave their written informed consent. The study was approved by the Ethics Committee on Human Research, Faculty of Medicine, Kocaeli University, Turkey (KOÜ KAEK 2015/113).

Right RA was used as the first choice throughout the study. Left RA was used in case of negative modified Allen's test, previous coronary artery bypass grafting, or previous use of right RA. After subcutaneous injection of prilocaine, RA was punctured with a $21 \mathrm{G}$ needle and a 0.021 ' guidewire. After skin incision, $10 \mathrm{~cm} 6 \mathrm{~F}$ hydrophilic coated sheath (Radifocus, Terumo Corp.) was introduced into the artery. Intra-arterial nitroglycerin $(200 \mu \mathrm{g})$ was injected through the sheath for spasm prophylaxis. Procedures were performed with $5 \mathrm{~F}$ diagnostic catheters (Optitorque, Terumo Corp.) or $6 \mathrm{~F}$ standard coronary guiding catheters (Launcher,
Medtronic Inc.). Unfractionated heparin (5000 IU) was injected through diagnostic catheter into the ascending aorta. RA spasm was defined as marked forearm pain with resistance to catheter movements or sheath removal. Hemostasis was achieved with a simple gauze and compression technique without any special equipment (such as inflatable wrist band, etc.). Procedure time was measured from the time of sheath insertion to sheath removal at the end of the procedure. RA occlusion was evaluated with Doppler examination and defined as the absence of antegrade flow in RA. Transradial procedures were performed by experienced interventional cardiologists who use RA as default arterial access site.

Ultrasound examinations were recorded at morning hours of the procedure day and follow-up day (at 1 week) in a quiet, temperature controlled and dim room. Ultrasound images were recorded on a hard drive of a high resolution ultrasound unit (Vivid S5, GE Health Care) with 4-13 MHz linear array probe. Patients were instructed to fast and abstain from caffeine, alcohol and tobacco for at least $12 \mathrm{~h}$ before the examination. Medical treatment of patients was not withheld before baseline and follow-up examinations. Patients rested in supine position for approximately $20 \mathrm{~min}$ before evaluation. Vascular probe was located $2-3 \mathrm{~cm}$ above puncture point for the evaluation of sheath injury.

Standard examination measurements were RA diameters and flow velocities at baseline, after brachial artery occlusion and after nitroglycerin administration before and 1 week after the procedure. Longitudinal images were preferred for the measurement of RA diameter. Flow velocities were acquired after necessary angle correction and with sample volume located at the center of artery. After baseline images were recorded, blood pressure cuff located above elbow was inflated to suprasystolic pressure for $5 \mathrm{~min}$. Highest hyperemic diameter and flow velocity was scanned between $30 \mathrm{~s}$ to 2 min after cuff deflation, which were generally observed after $45-60 \mathrm{~s}$. At least a 10 min break was kept between flow mediated dilatation (FMD) and nitrate mediated dilation (NMD) examinations. A single dose $(400 \mu \mathrm{g})$ of nitroglycerin in commercially available spray form was given orally. Highest diameter and flow velocity of RA was scanned between 3 and $4 \mathrm{~min}$ of nitroglycerin application. Ultrasound examinations were performed and analyzed offline by a single cardiologist blinded to clinical data of the patients. 


\section{Statistical analysis}

Continuous datawere presentedas means \pm standard deviations or medians (interquartile ranges) depending on normality of distribution. Paired samples t-test or Wilcoxon signed-rank test was used for comparison of pre- and post-procedural ultrasound findings. T-test or Mann-Whitney U test was used for comparison of continuous data of two separate populations. Categorical variables were presented as numbers (percentages) and compared with $\chi^{2}$ or Fisher's exact test. Correlations between two variables was tested with Pearson test. Logistic regression analysis was used to determine independent variables associated with RA injury. Results were tabulated as odds ratios (OR) and 95\% confidence intervals (CI). A two sided p value $<0.05$ was considered significant. Data collection and analysis were performed using Statistical Package for the Social Sciences, version 11.0 (SPSS Inc.).

\section{Results}

Fifty-two consecutive patients were prospectively included. One patient declined to attend follow-up visit and was excluded from analysis. The majority of procedures were performed through right RA (Table 1). There was only one access site crossover which was due to severe spasm; the patient was not excluded from the study. Two out of three procedures were completed with a single catheter. Again approximately two thirds of patients were found to have coronary artery disease.

At follow-up, 4 patients were found to have RA occlusion. These were considered RA-injured patients and included in the analysis as impaired vasodilatory function. Mean diameter of RA was $2.49 \mathrm{~mm}$ (range $1.7-3.2$ ) and $71 \%$ of participants had RA diameter smaller than outer diameter of $6 \mathrm{~F}$ sheath (Table 2). RA diameter significantly increased 1 week after transradial procedure, while the flow did not change significantly. In a similar manner, hyperemic and nitrate-mediate diameters increased significantly compared to pre-procedural findings and again flow velocities did not differ. Median FMD of the study was $15.3 \%$ and median NMD was $8.6 \%$. These values significantly decreased after 1 week.

Figure 1 represents the distribution of absolute change in FMD and NMD in study patients. Considering negative values a deterioration, $67 \%$ and $71 \%$ of study participants were found to have impaired RA vasodilatory function at 1 week. Alternatively, both FMD and NMD were impaired in
Table 1. Baseline data.

\begin{tabular}{|c|c|}
\hline \multicolumn{2}{|l|}{ Demographics } \\
\hline Age [years] & $62 \pm 11$ \\
\hline Male sex & $28(55 \%)$ \\
\hline Hypertension & $21(41 \%)$ \\
\hline Diabetes & $10(20 \%)$ \\
\hline Hyperlipidemia (\%) & $8(16 \%)$ \\
\hline Smoker [\%] & $6(12 \%)$ \\
\hline Weight [kg] & $75 \pm 12$ \\
\hline Height [m] & $1.63 \pm 0.07$ \\
\hline Body mass index $\left[\mathrm{kg} / \mathrm{m}^{2}\right]$ & $28.3 \pm 5.2$ \\
\hline Previous PCl & $7(14 \%)$ \\
\hline Previous CABG & $6(12 \%)$ \\
\hline \multicolumn{2}{|l|}{ Medications } \\
\hline Antiplatelet & $43(84 \%)$ \\
\hline ACEI & $9(18 \%)$ \\
\hline ARB & $5(10 \%)$ \\
\hline Beta-blocker & $34(67 \%)$ \\
\hline Calcium channel blocker & $2(3.9 \%)$ \\
\hline Diuretic & $4(7.8 \%)$ \\
\hline Statin & $13(26 \%)$ \\
\hline Oral anti-diabetic & $3(5.9 \%)$ \\
\hline Insulin & $3(5.9 \%)$ \\
\hline \multicolumn{2}{|l|}{ Procedural findings } \\
\hline Right radial artery & $44(86 \%)$ \\
\hline Diagnostic angiography & $40(78 \%)$ \\
\hline $\mathrm{PCl}$ & $11(22 \%)$ \\
\hline Procedure time [min] & $8.2(7-12)$ \\
\hline \multicolumn{2}{|l|}{ Number of catheters used: } \\
\hline 1 & $34(67 \%)$ \\
\hline 2 & $15(30 \%)$ \\
\hline 3 & $2(3.9 \%)$ \\
\hline Radial artery spasm & $7(14 \%)$ \\
\hline Access site crossover & $1(2.0 \%)$ \\
\hline \multicolumn{2}{|l|}{ Coronary artery disease: } \\
\hline Nonexistent & $18(35 \%)$ \\
\hline Single vessel disease & $19(37 \%)$ \\
\hline Multivessel disease & $14(28 \%)$ \\
\hline Radial artery occlusion & $4(7.8 \%)$ \\
\hline
\end{tabular}

ACEI - angiotensin converting enzyme inhibitor; ARB — angiotensin receptor blocker; CABG - coronary artery bypass grafting; $\mathrm{PCl}$ - percutaneous coronary intervention

$28(55 \%)$ patients, and either FMD or NMD was impaired in $42(82 \%)$ patients.

We compared patients with impaired vasodilatory functions with those with preserved vasodilatory functions (Table 3). Somewhat expectedly, 
Table 2. Ultrasound assessment of radial artery.

\begin{tabular}{lccc}
\hline & Pre-procedure & At 1 week & P \\
\hline Baseline diameter [mm] & $2.49 \pm 0.36$ & $2.84 \pm 0.30$ & $<0.001$ \\
Baseline flow [cm/s] & $68 \pm 20$ & $63 \pm 23$ & 0.065 \\
Hyperemia diameter [mm] & $2.84 \pm 0.41$ & $3.11 \pm 0.36$ & $<0.001$ \\
Hyperemia flow [cm/s] & $80 \pm 25$ & $81 \pm 28$ & 0.899 \\
Nitrate mediated diameter [mm] & $2.78 \pm 0.41$ & $3.05 \pm 0.37$ & $<0.001$ \\
Nitrate mediated flow [cm/s] & $72 \pm 21$ & $71 \pm 28$ & 0.668 \\
Flow mediated dilation [\%] & $15.3(5.9-18.5)$ & $10.3(3.4-16.1)$ & 0.019 \\
Nitrate mediated dilation [\%] & $8.6(4.5-17.9)$ & $6.9(3.3-11.5)$ & 0.005 \\
\hline
\end{tabular}
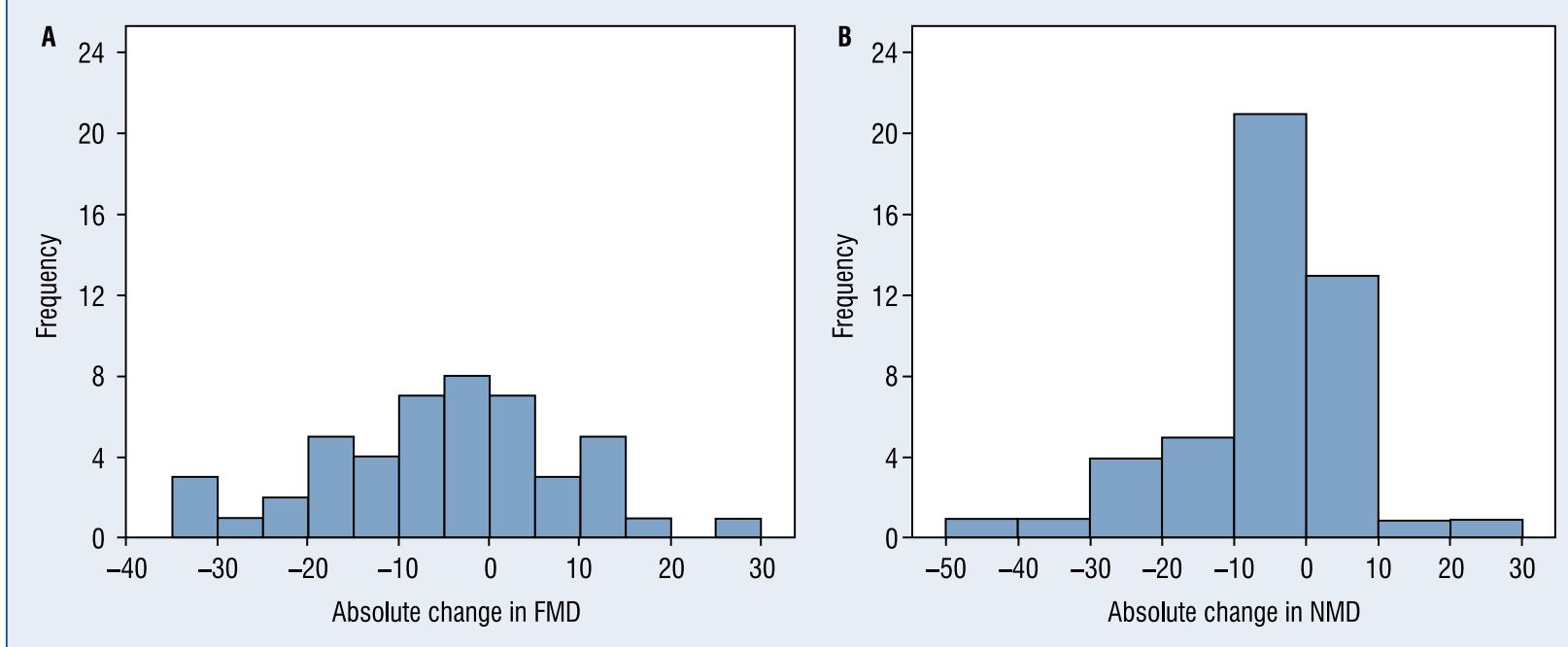

Figure 1. Distributions of absolute change in flow mediated dilation (FMD) (A) and nitrate mediated dilation (NMD) (B).

FMD was impaired more frequently in patients with relatively high pre-procedural values, however baseline RA diameter was not different between two groups. Impaired FMD was observed significantly less in patients using a renin-angiotensin system (RAS) inhibitor that is angiotensin converting enzyme inhibitor (ACEI) or angiotensin receptor blocker (ARB). Additionally, hypertension tended to be less frequent in impaired FMD group. Like FMD, NMD was impaired more frequently in patients with high pre-procedural values while RA diameter was marginally smaller in impaired NMD groups. Median procedure time was significantly longer in impaired group compared to the preserved group.

Logistic regression analyses were performed to find out independent predictors of deteriorating RA function. Both models included variables age, sex, body mass index, hypertension, diabetes, smoking, presence of coronary artery disease and those variables with a $\mathrm{p}$ value $<0.100$ in univariate analysis (RAS inhibitor use for FMD, procedure time and baseline RA diameter for NMD). Mean accuracy of the models were $77 \%$ and $75 \%$ for the impairment in FMD and NMD, respectively.

Multivariate analyses revealed that treatment with any RAS inhibitor was independently associated with less RA injury assessed with FMD (OR $0.241,95 \%$ CI $0.066-0.883, \mathrm{p}=0.032)$. On the other hand, smaller RA diameter (OR 0.079, 95\% CI $0.009-0.720, \mathrm{p}=0.024$ ) and potentially longer procedure time (OR 1.156, 95\% CI 0.989-1.350, $\mathrm{p}=0.068$ ) were associated with more RA injury assessed with NMD, though the latter had borderline statistical significance.

Effects of RAS blockade and baseline RA diameter on change in FMD and NMD were depicted in Figures 2 and 3 . There was a moderate but significant correlation between baseline RA diameter and absolute change in NMD $(\mathrm{r}=0.419, \mathrm{p}<0.001)$. 
Table 3. Comparison of patients with preserved and impaired vasodilation.

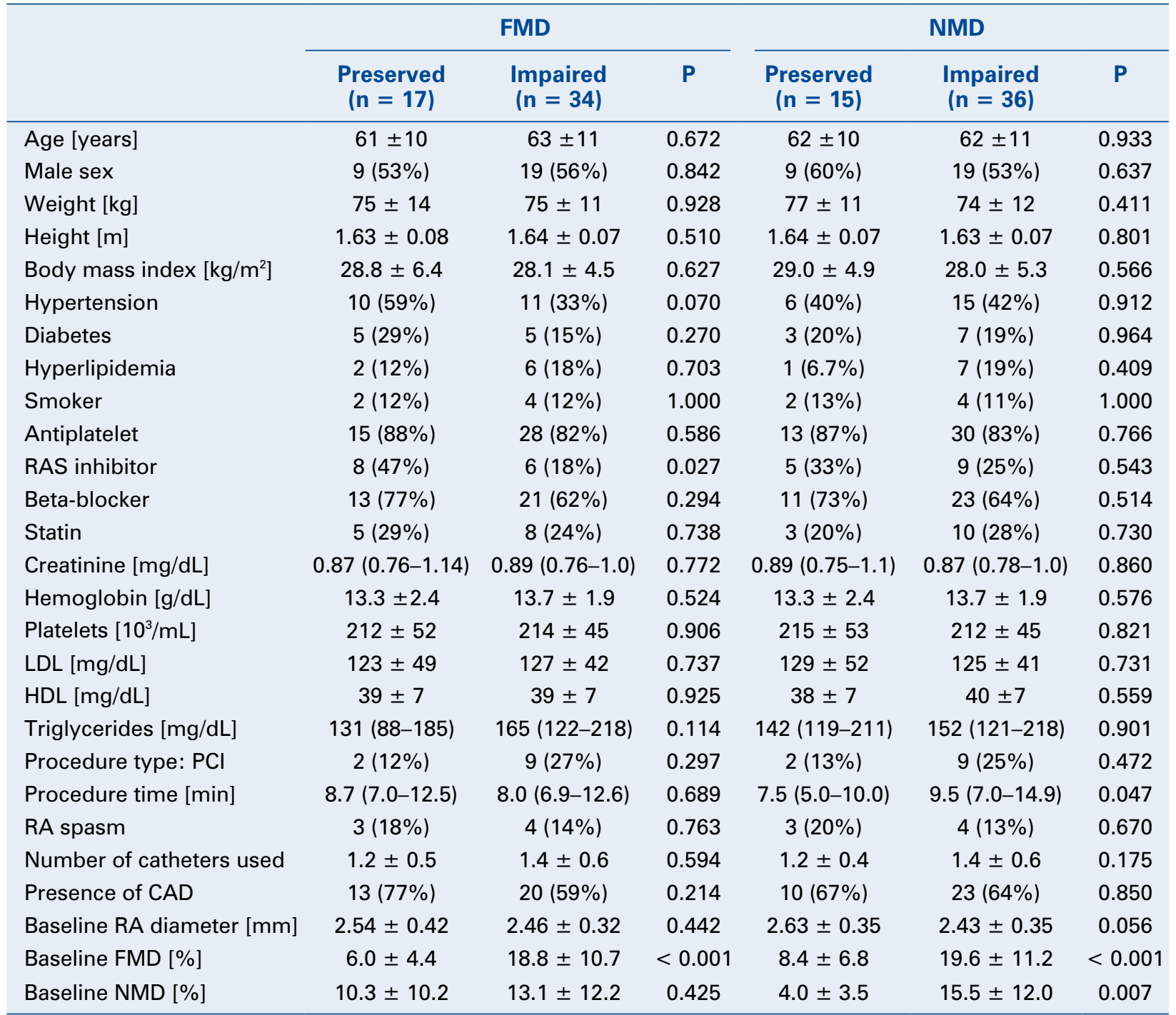

CAD — coronary artery disease; FMD — flow mediated dilation; HDL — high density lipoproteins; LDL — low density lipoproteins; NMD - nitrate mediated dilation; PCI — percutaneous coronary intervention; RA — radial artery; RAS - renin-angiotensin system

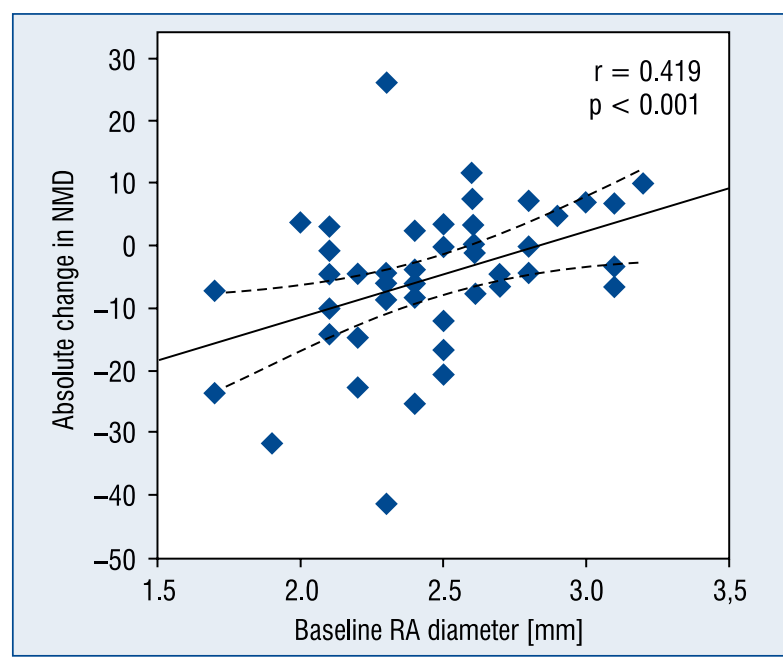

Figure 2. Scatter dot plot of change in nitrate mediated dilation (NMD) vs. baseline radial artery (RA) diameter.

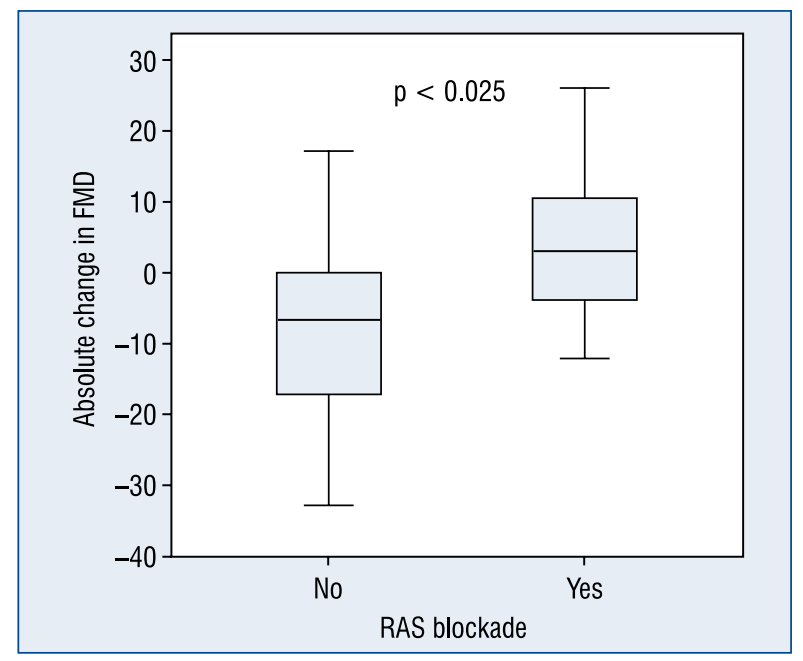

Figure 3. Comparison of change in flow mediated dilation (FMD) according to renin-angiotensin system (RAS) inhibitor use. 
Absolute change in FMD was significantly different in patients using a RAS inhibitor compared to those who were not $(1.9 \pm 12.9 \%$ vs. $-7.7 \pm 12.7 \%$, respectively, $\mathrm{p}=0.025)$. However, it must be noted that baseline FMD of patients using a RAS inhibitor was already lower than other patients $(4.3$ [0-10.3] vs. 16 [12.0-19.3] \%, p = 0.002) which may be related to higher incidence of hypertension (71.4 vs. $29.7 \%, p=0.007$ ). While FMD of patients on RAS inhibitors did not change significantly after 1 week (4.3 [0-10.3] vs. 10.3 [5.2-16.4] \%, p = 0.507), other patients experienced a significant reduction in FMD (16 [12.0-19.3] vs. 10.2 [3.4-15.7] \%, $\mathrm{p}=0.002)$.

\section{Discussion}

In our study of consecutive patients undergoing elective transradial procedure, we have confirmed that vasodilatory functions of RA were impaired after the procedure. However, functional impairment did not develop in all the patients. Actually, approximately two thirds of patients have shown impaired vasodilation whether it be assessed with FMD or NMD. More strikingly, only $55 \%$ of patients have shown impairment in both FMD and NMD. That means that not all patients were functionally impaired after transradial procedure, although we re-assessed patients at 1 week by which time some functional recovery might have occurred.

Flow mediated vasodilation is mediated by endothelium-derived nitric oxide in response to shear stress and is a measure of endothelial function. Endothelial dysfunction of RA possibly results from endothelial denudation injury as induced in animal models [15]. Complete or incomplete recovery of endothelial dysfunction is expected from as early as $24 \mathrm{~h}$ [16] up to 14 months after procedure $[6,9,12,17]$ although persistent impairment of RA function has also been reported [7, 10, 11, 13].

The major finding of our study was that deterioration of endothelial function following transradial catheterization was alleviated with the use of RAS inhibitors. ACEIs and ARBs improve endothelial function by inhibiting production of angiotensin II and downstream blockade of angiotensin II type 1 receptor, respectively. Angiotensin II plays a vital role in vasodilation [18]. By binding to type 1 receptor, angiotensin II leads to vasoconstriction, endothelial cell migration, proliferation and hypertrophy, increased uptake and oxidation of low density lipoprotein by endothelial cells and oxyradical production leading to endothelial dysfunction [19]. Additionally, increased bradykinin associated with angiotensin II inhibition increases the production and release of nitric oxide, prostacyclin and endothelium-derived hyperpolarizing factor via binding to B2 receptors [20-22). Both ACEIs and ARBs significantly improve endothelial dysfunction, determined by FMD, compared to placebo or other anti-hypertensive agents as reported by meta-analyses [23, 24], and difference between ACEIs and ARBs is not statistically significant. Our study was the first to report the benefits of RAS blockade in RA injury following transradial catheterization. Considering that hypertension is frequent in the general population and there is evidence-based benefit for ACEI therapy in patients with stable coronary artery disease even without hypertension, diabetes, left ventricular systolic dysfunction or chronic kidney disease [25], RAS blockade can have a clinical application in transradial approach. Having said that, unless RAS blockade is systematically studied with a randomized controlled trial in transradial approach, conclusion about its protective role should be noted with caution. That is due to the fact that we have also found that patients on RAS inhibitor therapy had lower baseline FMD compared to others which might be related to higher incidence of hypertension or undetected factors. As stated earlier, transradial catheterization impaired FMD of RA more severely in patients with higher baseline values than in patients with somewhat lower baseline values, expectedly. Therefore, benefit of RAS blockade may not be applicable to all patients.

Nitrate mediated vasodilation, on the other hand, is mediated by exogenous nitric oxide donor and is a measure of endothelium-independent vasodilation which is related to smooth muscle component of arterial system. Transradial catheterization disrupted NMD with a similar extent to FMD in our study. This effect on NMD has been consistently observed in previous studies as well [9-12], which means that transradial procedures traumatize not only endothelial layer but arterial wall as a whole. RA diameter and potential procedure time were associated with NMD impairment in this study. A similar finding has been reported recently, where pre-procedural RA diameter to sheath size ratio predicted NMD reduction, albeit after 6 months [11]. Approximately $71 \%$ of participants had RA diameter smaller than outer diameter of $6 \mathrm{~F}$ sheath in our study, so mechanical sheath injury was highly likely. Longer duration of exposure to a sheath larger than its internal diameter was obviously associated with impaired NMD of RA. As only $6 \mathrm{~F}$ sheath was used in our study, we could not evaluate 
the effect of sheath size. However, based on our findings and previous studies, downsizing transradial equipment as safely as possible will clearly be a sensible choice for the prevention of RA injury.

Importantly, transradial catheterization causes an increase in RA diameter in acute phase which can be explained by direct mechanical (dilating) injury to arterial wall. Like in balloon angioplasty, damage induced by dilation of RA may trigger the events that lead to intimal hyperplasia and vascular remodeling [26]. Observed in our study, as well as in a previous one [9], this fact actually constitutes a limitation to FMD and NMD calculations because increase in baseline RA diameter early after procedure inevitably decreases the proportion of the same absolute increase in diameter with exogenous stimuli.

\section{Limitations of the study}

Important limitations were discussed previously. Others are acknowledged here. Obviously, a larger study population could have revealed other possible predictors, such as hypertension, coronary artery disease, number of catheter changes, etc. Additionally, long-term ultrasound examination could find out the incidence of persistent injury or further recovery of vasodilatory function, if any. However, long-term effects of sheath injury are controversial and dependent on the time of assessment after procedure, as discussed previously [6].

\section{Conclusions}

Vasodilatory function of RA gets impaired in most patients early after transradial procedure. RAS blockade seems to exert a protective role against deteriorating endothelium-dependent vasodilation, whereas smaller RA diameter and potentially longer procedure time are associated with impaired endothelium-independent vasodilation.

\section{Conflict of interest: None declared}

\section{References}

1. Jolly SS, Yusuf S, Cairns J et al.; RIVAL trial group. Radial versus femoral access for coronary angiography and intervention in patients with acute coronary syndromes (RIVAL): A randomised, parallel group, multicentre trial. Lancet, 2011; 377: 1409-1420.

2. Wakeyama T, Ogawa $\mathrm{H}$, Iida $\mathrm{H}$ et al. Intima-media thickening of the radial artery after transradial intervention. An intravascular ultrasound study. J Am Coll Cardiol, 2003; 41: 1109-1114.

3. Staniloae CS, Mody KP, Sanghvi K et al. Histopathologic changes of the radial artery wall secondary to transradial catheterization. Vasc Health Risk Manag, 2009; 5: 527-532.

4. Yonetsu T, Kakuta T, Lee $\mathrm{T}$ et al. Assessment of acute injuries and chronic intimal thickening of the radial artery after transradial coronary intervention by optical coherence tomography. Eur Heart J, 2010; 31: $1608-1615$.
5. Edmundson A, Mann T. Nonocclusive radial artery injury resulting from transradial coronary interventions: Radial artery IVUS. J Invasive Cardiol, 2005; 17: 528-531.

6. Gaudino M, Leone A, Lupascu A et al. Morphological and functional consequences of transradial coronary angiography on the radial artery: Implications for its use as a bypass conduit. Eur J Cardiothorac Surg, 2015; 48: 370-374. doi: 10.1093/ejcts/ezu456.

7. Okuyan H, Açikgoz SK, Tacoy G, Kocaman SA, Abaci A. Effect of transradial coronary angiography procedure on vascular diameter and vasodilator functions in the access site. Angiology, 2013; 64: 515-521.

8. Park KH, Park WJ, Kim MK, et al. Effects of trimetazidine on endothelial dysfunction after sheath injury of radial artery. Am J Cardiol, 2010; 105: 1723-1727.

9. Sanmartin M, Goicolea J, Ocaranza R, Cuevas D, Calvo F. Vasoreactivity of the radial artery after transradial catheterization. J Invasive Cardiol, 2004; 16: 635-638.

10. Yan Z, Zhou Y, Zhao Y, Zhou Z, Yang S, Wang Z. Impact of transradial coronary procedures on radial artery function. Angiology, 2014; 65: 104-107.

11. Buturak A, Tekturk BM, Degirmencioglu A et al. Transradial catheterization may decrease the radial artery luminal diameter and impair the vasodilatation response in the access site at late term: An observational study. Heart Vessels, 2015; Feb 5, Epub ahead of print. doi: 10.1007/s00380-015-0640-x.

12. Dawson EA, Rathore S, Cable NT, Wright DJ, Morris JL, Green DJ. Impact of catheter insertion using the radial approach on vasodilatation in humans. Clin Sci (Lond), 2010; 118: 633-640.

13. Burstein JM, Gidrewicz D, Hutchison SJ, Holmes K, Jolly S, Cantor WJ. Impact of radial artery cannulation for coronary angiography and angioplasty on radial artery function. Am J Cardiol, 2007; 99: 457-459.

14. World Medical Association Declaration of Helsinki: Ethical Principles for Medical Research Involving Human Subjects. JAMA, 2013; 310: 2191-2194.

15. Berdeaux A, Ghaleh B, Dubois-Rande JL et al. Role of vascular endothelium in exercise-induced dilation of large epicardial coronary arteries in conscious dogs. Circulation, 1994; 89: 2799-2808.

16. Heiss C, Balzer J, Hauffe T et al. Vascular dysfunction of brachial artery after transradial access for coronary catheterization: Impact of smoking and catheter changes. J Am Coll Cardiol Cardiovasc Interv 2009; 2: 1067-1073.

17. Madssen E, Haere P, Wiseth R. Radial artery diameter and vasodilatory properties after transradial coronary angiography. Ann Thorac Surg, 2006; 82: 1698-1702.

18. Watanabe T, Barker TA, Berk BC. Angiotensin II and the endothelium: Diverse signals and effects. Hypertension, 2005; 45: 163-169.

19. Bell L, Madri JA. Influence of the angiotensin system on endothelial and smooth muscle cell migration. Am J Pathol, 1990; 137: 7-12.

20. Barrow SE, Dollery CT, Heavey DJ, Hickling NE, Ritter JM, Vial J. Effect of vasoactive peptides on prostacyclin synthesis in man. $\mathrm{Br}$ J Pharmacol, 1986; 87: 243-247.

21. O'Kane KP, Webb DJ, Collier JG, Vallance PJ. Local L-NG-monomethyl-arginine attenuates the vasodilator action of bradykinin in the human forearm. Br J Clin Pharmacol, 1994; 38: 311-315.

22. Mombouli JV, Illiano S, Nagao T, Scott-Burden T, Vanhoutte PM. Potentiation of endothelium-dependent relaxations to bradykinin by angiotensin I converting enzyme inhibitors in canine coronary artery involves both endothelium-derived relaxing and hyperpolarizing factors. Circ Res, 1992; 71: 137-144.

23. Shahin Y, Khan JA, Samuel N, Chetter I. Angiotensin converting enzyme inhibitors effect on endothelial dysfunction: A meta-analysis of randomised controlled trials. Atherosclerosis, 2011; 216: 7-16.

24. Li S, Wu Y, Yu G, Xia Q, Xu Y. Angiotensin II receptor blockers improve peripheral endothelial function: A meta-analysis of randomized controlled trials. PLoS One, 2014; 9: e90217.

25. Fihn SD, Gardin JM, Abrams J et al. 2012 ACCF/AHA/ACP/AATS/ /PCNA/SCAI/STS Guideline for the diagnosis and management of patients with stable ischemic heart disease: A report of the American College of Cardiology Foundation/American Heart Association Task Force on Practice Guidelines, and the American College of Physicians, American Association for Thoracic Surgery, Preventive Cardiovascular Nurses Association, Society for Cardiovascular Angiography and Interventions, and Society of Thoracic Surgeons. J Am Coll Cardiol, 2012; 60: e44-e164.

26. Mintz GS, Popma JJ, Pichard AD et al. Arterial remodeling after coronary angioplasty: A serial intravascular ultrasound study. Circulation, 1996; 94: 35-43. 